\title{
Establishing Normative Data for 10RM Strength Scores in College- Aged Males
}

\author{
Timothy J. Piper ${ }^{1}$, Seth M. Furman², Thomas J. Smith ${ }^{3}$ and Michael A. Waller ${ }^{4}$.
}

${ }^{1}$ Department of Kinesiology, Western Illinois University, ${ }^{2}$ Exercise Science, Salisbury University, ${ }_{3}$ Educational Technology, Research and Assessment, Northern Illinois University, ${ }^{4}$ Health and Physical Education, Arkansas Tech University.

\section{ABSTRACT}

Training recommendations for novice to intermediate lifters include loads that correspond to a 10RM, yet there has not been normative data established for college aged males. Therefore, the purpose of this study was to provide 10RM normative reference values for various strength exercises for 18 to 25-year-old males. The exercises included in the study were the Lat Pulldown, Bench Press, Seated Front Press, Preacher Curl, and the Leg Press. All testing and training occurred in the same facility and utilized the same equipment for testing and training. Testing occurred prior to the structured training program began and then again upon completion of 12 weeks of training. A total of 1095 subjects $($ age $=19.94+1.633$ years; height $=70.57+3.017$ inches; pre-test bodyweight $=188.30+40.975$ pounds; post-test bodyweight $=190.31+40.688$ pounds; years of strength training experience = $3.76+2.484$ years, ranging from zero experience up to 13 years) participated in the study. Bodyweight categories were derived based upon two established classification systems used in competitive lifting sports. Percentiles and performance rankings for each weight category were reported, where the weighted average method was used to determine the percentile break points. These norms provide a range of possible 10RM loads as well as a reference to the strength levels, which could be useful for fitness professionals to more effectively assess and design resistance training programs.

Key Words: Leg Press, Bench Press, Lat Pulldown, Bicep Curl, Seated Overhead Press.

\section{INTRODUCTION}

Muscular strength, defined as the ability to exert force on an external object or resistance (28, 31 ), is a fitness component and promoted as an essential part of athletic performance. A lack of muscular strength makes activities of daily living more difficult as individuals age, while upper body and lower body strength has been suggested as a predictor of mortality in older men and women respectively (24). Low levels of muscular strength are associated with health problems and functional limitations (5), as there is an inverse relationship between strength and metabolic syndrome (19, 20). The importance of muscular strength is an established necessity for sport performance as it is a critical factor for the presentation of maximal speed, power, force, agility, and injury risk reduction (32). The general healthy adult population (> 18 years) is recommended to begin resistance training programs with loads that can be achieved for 8-12 repetitions to improve muscular strength $(2,25)$. Normative values have been compiled for a wide array of resistance training exercises for multiple age groups, and populations. The Cooper Institute is one of the most commonly referenced sources of normative data, while the American College of Sports Medicine has published weight training exercise recommendations and program requirements (2, 4, $12,17,26)$.Although these recommendations exist, much of this normative and referenced data are based upon small sample sizes or were produced in the 1990's which most likely no longer represent current fitness levels for specific populations. Fitness practitioners need current information for comparison of their clientele when designing a strength program. The development of normative 
data, and inclusion of an expected objective measure of strength could enhance program design and efficacy. Using a 10-repetition maximum (10RM) allows for the load to be submaximal and may provide a better representation of the loads more commonly prescribed for the novice client. Researchers have conducted studies using the 10RM for the purpose of assessing changes hypertrophy, strength, power, and hormones $(13,15,18,23)$. While the 10RM is commonly used for research to date no study has been conducted with the purpose of establishing 10RM normative data for college aged men. The establishment of 10RM normative data would be useful for fitness professionals to more effectively design resistance training programs. Therefore, the purpose of this study is to provide 10RM normative reference values for various strength exercises for 18 to 25-year-old males.

\section{METHODS}

\section{Experimental Approach to the Problem}

Cross-sectional performance and anthropometric data were obtained over the course of five years as part of a college basic strength training course. Data and training were conducted by twelve graduate students who were under the direct supervision and training from a National Strength and Conditioning Association (NSCA) Certified Strength and Conditioning Specialist (CSCS). All exercises were performed according to the guidelines established by the NSCA $(4,14)$. All testing and training occurred in the same facility and utilized the same equipment for testing and training. Testing occurred prior to the structured training program began and then again upon completion of 12 weeks of training. All data from these strength tests were analyzed using IBM SPSS version 24. To establish bodyweight categories, we made use of two established classifications: the World Drug Free Powerlifting Federation (WDFPF) weight classes and the International Weightlifting Federation (IWF) weight classes. These derived weight categories were: $135 \mathrm{lbs}$. or less, greater than 135 lbs. to 150 lbs., 150-1-165, 165.1-190, 190.1210, 210.1-240, 240.1-270, greater than 270.

\section{Subjects}

Subjects included a sample of $N=1095$ of male, college students. The Western Illinois University Institutional Review Board (IRB) in the Office of Sponsored Projects approved the study.

\section{Operational definition of 10RM}

To establish consistency with test results an operational definition of 10RM was necessary. For the purpose of this study a 10RM is defined as: the most weight that could be lifted for 10 repetitions with good technique. The aim of each test set was not to reach a point of complete failure but rather the highest load possible while maintaining proper form on each repetition. Speed of movement was not controlled but participants were encouraged to complete the concentric phase as quickly as possible and control the eccentric phase of each repetition. For all testing sessions, any test sets that did not follow the NSCA established lifting technique guidelines $(4,14)$ were deemed invalid and resulted in those subjects re-testing for the given exercise until they achieved a 10RM with the required technique.

\section{Procedures}

Prior to each testing session, subjects performed a general total body warm-up by performing five to 10 minutes of calisthenics, in addition to dynamic and static stretches. After the warm-up, subjects were tested on each of the exercises, with the exercises performed in random order. Exercises were performed according to the guidelines established by the $\operatorname{NSCA}(4,14)$. All exercises were demonstrated and then supervised by researchers under the direct supervision and training of the primary investigator, who holds the title of NSCA-CSCS*D. This strict adherence to the NSCA guidelines was maintained to ensure that the specifications of the testing battery and exercise technique were properly followed. Any technique modifications are indicated with each respective exercise. Verbal encouragement was provided during each testing attempt. Spotters were available for lift-offs and for any needed assistance for safety purposes. Any test attempt in which spotters assisted in the performance of the exercise were considered unsuccessful and were not included in the data analysis.

For each test performed, subjects were instructed to self-select initial loads that they were confident they could perform for five to eight repetitions. After they completed one set with their starting load, they rested for approximately three minutes, and then performed another set with more weight. This procedure was repeated for at least five sets, but not more than eight sets. When subjects felt they had found a load they perceived was the most they could lift for 10 repetitions with good technique, they were directly observed by at least one researcher 
who evaluated each test attempt to ensure that lifting techniques met the prescribed requirements. If test sets were evaluated to be too heavy or were performed with poor technique, subjects were instructed to rest, lower the weight, and perform another test attempt. If the test was successful but deemed to be under the true 10RM, subjects were instructed to rest, add weight, and perform another test attempt. These procedures were repeated until a 10RM was achieved for each strength measure.

\section{Training Protocol}

The entire program consisted of 2 weeks of pretesting, 12 weeks of structured training, and concluded with 2 weeks of post-testing. Daily training sessions were led by NSCA-CSCS professors and trained graduate assistants who taught each exercise technique according to NSCA guidelines $(4,14)$. Each 50-minute training session included at least two multi-joint exercises for variations of squatting, pushing, and pulling exercises for a total of 18 sets minimum per session. Each session was concluded with some form of abdominal exercises for 1-3 sets of 10-20 reps or 30-60 seconds.

Daily workouts were recorded on training logs that were monitored for adherence and effort. This close monitoring helped to ensure that participants were compliant with total-body training requirements.

The set and rep scheme followed a traditional periodization model using three sets per exercise of varying RM loads. The first 6 weeks consisted of three sets of 8-10RM loads, followed by three weeks of three sets of 5RM loads, and concluded with three weeks of three sets of 3RM loads. Regardless of which rep scheme was being performed, sets one and two acted as warm-up sets prior to the most intense third set. Loads were adjusted daily to levels that allowed each exercise to be performed with proper technique up to, but not surpassing, a repetition maximum (RM) load on the final set.

\section{Lat Pulldown}

A lat pulldown station on a multi-station machine was used for all lat pulldown testing, (MuscleMaxx, multi-station compact gym).

\section{Bench Press}

The equipment used for bench press testing included a Legend 3-way adjustable bench (model 3103), a 45 lbs. York barbell, and York iron pound weight plates.

\section{Seated Barbell Shoulder Press}

The equipment used for seated front press testing included a Legend 3-way adjustable bench (model 3103), a 45-pound York barbell, and York iron pound weight plates. The seat back was inclined to 750 while subjects were seated in the five-point body contact position. The procedures for this lift were modified from the technique described in the literature (8), adopted under advisement of school athletic training staff to decrease the stress on the acromioclavicular joint. The modification included lowering the barbell in front of the face until it was immediately below the chin instead of to the clavicles.

\section{Preacher Curl}

The preacher curl was performed on a seated preacher curl station (Body Masters BE 207) with the arm pad angle of $40^{\circ}$, a standard 22-pound EZ curl barbell (York Barbell Olympic EZ curl bar, model 32042) and York iron pound weight plates. This exercise protocol was specifically developed for this study with collaboration from the CSCS researchers and athletic training staff. Subjects were seated on the bench with both feet flat on the floor. The height of the preacher bench pad was adjusted so that the posterior aspect of the upper arms rested flat on the arm pad. Using a supinated grip, subjects grasped the curling bar with the webbing of the thumbs resting in the narrowest portion of the curved portion of the EZ curl bar, with the hands between seven and 10 inches apart depending on hand size. The hands were held in approximately 250 of supination in line with the angle of the barbell. Subjects started in a fully flexed position (A point of contact between the forearms and biceps brachii). Once starting positioned was assumed, subjects then performed the eccentric portion of the exercise until the elbow joints reached a position of approximately 5-10 degrees of flexion in the elbow as measured by a goniometer (this procedure was adopted under advisement of the IRB and school athletic training staff to decrease the stress on the elbow joint). Subjects lifted the bar upward to the starting position by contracting the elbow flexors until the elbow joints were fully flexed while maintaining foot contact with the floor and buttocks in contact with the bench pad. No forward torso movement or backward rocking was allowed throughout the exercise. 
Leg Press

The equipment used for leg press testing included a $45^{\circ}$ leg press (ProMaxima, model P-118, height 63" Width-68", length 60") and York iron pound weight plates.

\section{Statitical Analysis}

IBM SPSS Statistics version 24 for Windows (IBM corporation, Armonk, New York) was used for statistical analysis. Within each age group, extreme values (either very low or very high values) that exceeded $2 \times$ IQR were Winsorized. These extreme values were few in number (three cases for pretest data, nine cases for posttest data), were primarily high values (83\%) as opposed to low values (17\%), and the majority (58\%) of these extreme values occurred with the preacher curl. A paired-samples t-test was used to determine whether there was a statistically significant mean difference between the pre-training and post-training strength measures. Further exploratory analysis of percentage increase for each lift was also calculated.

To generate standards for each strength measure, the 5th, 10th, 20th, 30th, 40th, 50th, 60th, 70th, 80th, 90th, and 95th percentiles (27) were reported, where the weighted average method (29) was used to determine the percentile break points.

\section{RESULTS}

The descriptive statistics can be found in Table 1.

Table 1. Descriptive statistics of entire sample

\begin{tabular}{|c|c|c|c|c|c|}
\hline & $\mathbf{N}$ & Minimum & Maximum & Mean & Std. Deviation \\
\hline Age & 1095 & 18 & 25 & 19.94 & 1.633 \\
\hline $\begin{array}{c}\text { Years of } \\
\text { Experience }\end{array}$ & 1030 & 0 & 13 & 3.76 & 2.484 \\
\hline Pre-test Height & 1094 & 57 & 83 & 70.57 & 3.017 \\
\hline $\begin{array}{c}\text { Pre-test } \\
\text { Bodyweight }\end{array}$ & 1077 & 101 & 387 & 188.30 & 40.975 \\
\hline $\begin{array}{c}\text { Post-test } \\
\text { Bodyweight }\end{array}$ & 1063 & 100 & 407 & 190.31 & 40.688 \\
\hline $\begin{array}{c}\text { Valid N } \\
\text { (listwise) }\end{array}$ & 980 & & & & \\
\hline
\end{tabular}

Table 2. Pre-training weight (lbs) categories

\begin{tabular}{|c|c|c|c|c|}
\hline & & Frequency & Percent & Valid Percent \\
\hline Valid & 135 or less & 50 & 4.6 & 4.7 \\
\hline & $135.1-150$ & 103 & 9.4 & 9.6 \\
\hline & $150.1-165$ & 188 & 17.2 & 17.5 \\
\hline & $165.1-190$ & 302 & 27.6 & 28.1 \\
\hline & $190.1-210$ & 177 & 16.2 & 16.5 \\
\hline & $210.1-240$ & 146 & 13.3 & 13.6 \\
\hline & $240.1-270$ & 63 & 5.8 & 5.9 \\
\hline & 270.1 or more & 46 & 4.2 & 4.3 \\
\hline & Total & 1075 & 98.2 & 100.0 \\
\hline & System & 20 & 1.8 & \\
\hline Missing & & 1095 & 100.0 & \\
\hline Total & & &
\end{tabular}


Table 3. Post-training weight (lbs) categories

\begin{tabular}{|c|c|c|c|c|}
\hline & & Frequency & Percent & Valid Percent \\
\hline Valid & 135 or less & 36 & 3.3 & 3.4 \\
\hline & $135.1-150$ & 92 & 8.4 & 8.7 \\
\hline & $150.1-165$ & 177 & 16.2 & 16.7 \\
\hline & $165.1-190$ & 324 & 29.6 & 30.6 \\
\hline & $190.1-210$ & 162 & 14.8 & 15.3 \\
\hline & $210.1-240$ & 152 & 13.9 & 14.3 \\
\hline & $240.1-270$ & 67 & 6.1 & 6.3 \\
\hline & 270.1 or more & 50 & 4.6 & 4.7 \\
\hline & Total & 1060 & 96.8 & 100.0 \\
\hline Missing & System & 35 & 3.2 & \\
\hline Total & & 1095 & 100.0 & \\
\hline
\end{tabular}

Tables 2 and 3 indicate the frequency of subjects, percentage of total, valid percentage of total subjects in each weight category for the pre-test and post-test respectively.

Upon further exploration the expected increases in strength for each exercise was found to vary for each exercise. T-test results revealed that each lift comparison pre-training to post-training scores all possessed a statistically significant increase ( $p>.001$ ), are displayed in table 4 , and include the mean score+standard deviation, and approximate percentage increase. The percentage increase between pre- and post-training was calculated by dividing the increase score by the original score and multiplying by 100 .

Percentile ranks, performance ranks, sample size (n), mean, and standard deviation for pre-training and post-training for each weight class for the lat pulldown, bench press, seated front press, preacher curl, and leg press, are reported in tables 5-14, respectively.

To further enhance the utility of the data performance rankings were determined on both the pre-training and post-training data. The methodology for determining the normative data performance rankings was adopted from prior research (30) and is as follows: Excellent $=$ Highest $5 \%$, Good $=$ next highest $15 \%$, Regular $=$ middle $60 \%$, Poor=next lowest $15 \%$, Very Poor $=$ next lowest $5 \%$. The rankings are displayed using gradients of grayscale highlights for each level of performance within tables 5-14. By designating the normative data with performance rankings practitioners can better explain an athlete's current strength level compared to this sample of college-aged students. This method of performance ranking is a common practice with a variety of other fitness measures and can be found in numerous resources $(4,9,12,17)$.

Table 4. Pre-training and post training comparisons, (all scores in pounds)

\begin{tabular}{|c|c|c|c|}
\hline & $\begin{array}{l}\text { Pre-Training Score } \\
\text { Mean } \pm \text { Std. Deviation }\end{array}$ & $\begin{array}{l}\text { Post-Training Score } \\
\text { Mean } \pm \text { Std. Deviation }\end{array}$ & $\begin{array}{l}\% \text { increase between } \\
\text { pre- and post-training }\end{array}$ \\
\hline Lat Pulldown & $170.80 \pm 29.82$ & $188.89 \pm 26.41$ & $10.6 \%$ \\
\hline Bench Press & $184.45 \pm 51.17$ & $204.01 \pm 52.85$ & $10.6 \%$ \\
\hline Seated Front Press & $123.32 \pm 30.52$ & $138.25 \pm 32.49$ & $12.1 \%$ \\
\hline Preacher Curl & $85.50 \pm 28.98$ & $98.18 \pm 25.90$ & $14.8 \%$ \\
\hline Leg Press & $482.12 \pm 190.14$ & $572.52 \pm 162.77$ & $18.8 \%$ \\
\hline
\end{tabular}


Table 5. Percentile ranks and descriptive statistics for Lat Pulldown Pre-Training

\begin{tabular}{|c|c|c|c|c|c|c|c|c|}
\hline \multicolumn{7}{|c|}{ Body Weight Class (Ibs) } \\
\hline Percentile & $\mathbf{< 1 3 5}$ & $\mathbf{1 3 5 < - 1 5 0}$ & $\mathbf{1 5 0}<-\mathbf{1 6 5}$ & $\mathbf{1 6 5}<-\mathbf{1 9 0}$ & $\mathbf{1 9 0 < - 2 1 0}$ & $\mathbf{2 1 0 < - 2 4 0}$ & $\mathbf{2 4 0 < - 2 7 0}$ & $\mathbf{2 7 0}$ \\
\hline $\mathbf{9 5 \%}$ & 134.50 & 158.00 & 160.00 & 170.00 & 200.00 & 200.00 & 189.00 & 200.00 \\
\hline $\mathbf{9 0 \%}$ & 130.00 & 140.00 & 160.00 & 160.00 & 170.00 & 180.00 & 180.00 & 173.00 \\
\hline $\mathbf{8 0 \%}$ & 120.00 & 130.00 & 140.00 & 150.00 & 160.00 & 160.00 & 160.00 & 163.00 \\
\hline $\mathbf{7 0 \%}$ & 120.00 & 120.00 & 140.00 & 140.00 & 150.00 & 150.00 & 160.00 & 150.00 \\
\hline $\mathbf{6 0 \%}$ & 113.00 & 120.00 & 130.00 & 140.00 & 140.00 & 150.00 & 140.00 & 150.00 \\
\hline $\mathbf{5 0 \%}$ & 110.00 & 110.00 & 120.00 & 130.00 & 140.00 & 140.00 & 130.00 & 150.00 \\
\hline $\mathbf{4 0 \%}$ & 104.00 & 110.00 & 120.00 & 120.00 & 130.00 & 130.00 & 130.00 & 140.00 \\
\hline $\mathbf{3 0 \%}$ & 100.00 & 110.00 & 110.00 & 120.00 & 130.00 & 120.00 & 120.00 & 130.00 \\
\hline $\mathbf{2 0 \%}$ & 90.00 & 100.00 & 100.00 & 110.00 & 120.00 & 120.00 & 120.00 & 120.00 \\
\hline $\mathbf{1 0 \%}$ & 80.00 & 90.00 & 100.00 & 100.00 & 107.00 & 106.00 & 110.00 & 110.00 \\
\hline $\mathbf{5 \%}$ & 70.00 & 80.00 & 90.00 & 100.00 & 100.00 & 100.00 & 100.00 & 100.00 \\
\hline $\mathbf{n}$ & 50 & 103 & 187 & 299 & 176 & 145 & 63 & 46 \\
\hline Mean & 107.20 & 114.93 & 124.89 & 131.67 & 139.72 & 139.63 & 139.92 & 143.80 \\
\hline SD & 18.22 & 20.65 & 23.15 & 22.79 & 27.37 & 27.95 & 26.24 & 25.85 \\
\hline
\end{tabular}

Table 6. Percentile ranks and descriptive statistics for Lat Pulldown Post-Training

\begin{tabular}{|c|c|c|c|c|c|c|c|c|}
\hline \multicolumn{10}{|c|}{ Body Weight Class (Ibs) } \\
\hline Percentile & $\mathbf{< 1 3 5}$ & $\mathbf{1 3 5 < - 1 5 0}$ & $\mathbf{1 5 0}<-\mathbf{1 6 5}$ & $\mathbf{1 6 5}<-\mathbf{1 9 0}$ & $\mathbf{1 9 0 < - 2 1 0}$ & $\mathbf{2 1 0 < - 2 4 0}$ & $\mathbf{2 4 0 < - 2 7 0}$ & $\mathbf{2 7 0}$ \\
\hline $\mathbf{9 5 \%}$ & 160.00 & 160.00 & 180.00 & 184.00 & 200.00 & 220.00 & 200.00 & 220.00 \\
\hline $\mathbf{9 0 \%}$ & 150.00 & 150.00 & 170.00 & 180.00 & 190.00 & 200.00 & 190.00 & 200.00 \\
\hline $\mathbf{8 0 \%}$ & 140.00 & 148.00 & 160.00 & 170.00 & 175.00 & 180.00 & 180.00 & 190.00 \\
\hline $\mathbf{7 0 \%}$ & 140.00 & 140.00 & 159.50 & 160.00 & 170.00 & 170.00 & 170.00 & 190.00 \\
\hline $\mathbf{6 0 \%}$ & 130.00 & 130.00 & 150.00 & 160.00 & 160.00 & 170.00 & 160.00 & 180.00 \\
\hline $\mathbf{5 0 \%}$ & 130.00 & 130.00 & 140.00 & 150.00 & 160.00 & 160.00 & 150.00 & 170.00 \\
\hline $\mathbf{4 0 \%}$ & 120.00 & 130.00 & 140.00 & 140.00 & 150.00 & 150.00 & 150.00 & 160.00 \\
\hline $\mathbf{3 0 \%}$ & 120.00 & 125.00 & 130.00 & 140.00 & 150.00 & 150.00 & 150.00 & 150.00 \\
\hline $\mathbf{2 0 \%}$ & 110.00 & 120.00 & 130.00 & 130.00 & 140.00 & 140.00 & 140.00 & 150.00 \\
\hline $\mathbf{1 0 \%}$ & 96.00 & 110.00 & 120.00 & 124.00 & 130.00 & 130.00 & 130.00 & 140.00 \\
\hline $\mathbf{5 \%}$ & 88.00 & 100.00 & 110.00 & 120.00 & 130.00 & 125.50 & 130.00 & 130.00 \\
\hline $\mathbf{n}$ & 35 & 91 & 176 & 323 & 159 & 150 & 67 & 49 \\
\hline Mean & 125.29 & 131.92 & 144.86 & 150.85 & 159.52 & 162.23 & 159.01 & 170.51 \\
\hline $\mathbf{S D}$ & 19.21 & 16.53 & 21.84 & 20.88 & 22.64 & 25.94 & 21.55 & 25.38 \\
\hline
\end{tabular}

\section{Index to Performance Rankings}

Excellent $=$ highest 5\%, Good= next highest 15\%, Average $=$ middle $60 \%$,

Below average=next lowest 15\%, and Poor=lowest 5\% 
Table 7. Percentile ranks and descriptive statistics for Bench Press Pre-Training

\begin{tabular}{|c|c|c|c|c|c|c|c|c|}
\hline \multicolumn{9}{|c|}{ Body Weight Class (Ibs) } \\
\hline Percentile & $<135$ & $135<-150$ & $150<-165$ & $165<-190$ & $190<-210$ & $210<-240$ & $240<-270$ & $>270$ \\
\hline $95 \%$ & 137.25 & 164.00 & 181.50 & 205.00 & 225.00 & 233.75 & 223.00 & 270.00 \\
\hline $90 \%$ & 135.00 & 145.00 & 165.00 & 185.00 & 218.00 & 225.00 & 205.00 & 235.00 \\
\hline $80 \%$ & 125.00 & 135.00 & 155.00 & 165.00 & 195.00 & 185.00 & 185.00 & 205.00 \\
\hline $70 \%$ & 115.00 & 135.00 & 144.50 & 155.00 & 185.00 & 185.00 & 175.00 & 190.00 \\
\hline $60 \%$ & 105.00 & 135.00 & 135.00 & 145.00 & 165.00 & 165.00 & 155.00 & 185.00 \\
\hline $50 \%$ & 95.00 & 115.00 & 135.00 & 135.00 & 155.00 & 155.00 & 155.00 & 160.00 \\
\hline $40 \%$ & 95.00 & 105.00 & 115.00 & 135.00 & 144.00 & 145.00 & 135.00 & 155.00 \\
\hline $30 \%$ & 95.00 & 98.00 & 115.00 & 135.00 & 135.00 & 135.00 & 135.00 & 135.00 \\
\hline $20 \%$ & 81.00 & 95.00 & 105.00 & 115.00 & 135.00 & 120.00 & 123.00 & 130.00 \\
\hline $10 \%$ & 65.00 & 85.00 & 95.00 & 95.00 & 115.00 & 115.00 & 97.00 & 95.00 \\
\hline $5 \%$ & 50.50 & 75.50 & 86.75 & 85.50 & 103.50 & 95.00 & 87.00 & 87.50 \\
\hline $\mathrm{n}$ & 50 & 101 & 186 & 301 & 176 & 144 & 63 & 44 \\
\hline Mean & 101.40 & 116.98 & 129.14 & 141.11 & 159.66 & 158.96 & 153.25 & 168.07 \\
\hline SD & 27.16 & 25.86 & 29.35 & 33.31 & 37.83 & 41.60 & 37.88 & 52.19 \\
\hline
\end{tabular}

Table 8. Percentile ranks and descriptive statistics for Bench Press Post-Training

\begin{tabular}{|c|c|c|c|c|c|c|c|c|}
\hline \multicolumn{9}{|c|}{ Body Weight Class (Ibs) } \\
\hline Percentile & $<135$ & $135<-150$ & $150<-165$ & $165<-190$ & $190<-210$ & $210<-240$ & $240<-270$ & $>270$ \\
\hline $95 \%$ & 156.00 & 166.00 & 196.50 & 225.00 & 225.00 & 239.50 & 225.00 & 295.00 \\
\hline $90 \%$ & 149.00 & 155.00 & 185.00 & 205.00 & 225.00 & 230.00 & 211.50 & 235.00 \\
\hline $80 \%$ & 139.00 & 150.00 & 170.00 & 185.00 & 205.00 & 225.00 & 205.00 & 225.00 \\
\hline $70 \%$ & 131.00 & 140.00 & 165.00 & 180.00 & 190.00 & 205.00 & 195.00 & 215.00 \\
\hline $60 \%$ & 120.00 & 135.00 & 155.00 & 170.00 & 185.00 & 195.00 & 185.00 & 205.00 \\
\hline $50 \%$ & 115.00 & 125.00 & 150.00 & 160.00 & 175.00 & 185.00 & 175.00 & 195.00 \\
\hline $40 \%$ & 109.00 & 120.00 & 145.00 & 155.00 & 165.00 & 170.00 & 155.00 & 185.00 \\
\hline $30 \%$ & 105.00 & 115.00 & 135.00 & 145.00 & 155.00 & 155.00 & 155.00 & 175.00 \\
\hline $20 \%$ & 95.00 & 115.00 & 130.00 & 135.00 & 145.00 & 145.00 & 147.00 & 155.00 \\
\hline $10 \%$ & 83.00 & 105.00 & 113.50 & 120.00 & 125.00 & 135.00 & 135.00 & 125.00 \\
\hline $5 \%$ & 69.00 & 95.00 & 103.50 & 115.00 & 115.00 & 117.75 & 118.50 & 112.50 \\
\hline $\mathbf{n}$ & 35 & 91 & 176 & 323 & 159 & 150 & 66 & 49 \\
\hline Mean & 116.14 & 129.73 & 150.03 & 162.35 & 173.71 & 181.63 & 174.47 & 192.14 \\
\hline SD & 23.89 & 21.28 & 28.73 & 32.33 & 35.56 & 38.86 & 31.73 & 47.68 \\
\hline
\end{tabular}

\section{Index to Performance Rankings}

Excellent $=$ highest $5 \%$, Good $=$ next highest 15\%, Average $=$ middle $60 \%$,

Below average =next lowest 15\%, and Poor=lowest 5\% 
Table 9. Percentile ranks and descriptive statistics for Seated Front Press Pre-Training

\begin{tabular}{|c|c|c|c|c|c|c|c|c|}
\hline \multicolumn{7}{|c|}{ Body Weight Class (Ibs) } \\
\hline Percentile & $\mathbf{< 1 3 5}$ & $\mathbf{1 3 5}<-\mathbf{1 5 0}$ & $\mathbf{1 5 0}<-\mathbf{1 6 5}$ & $\mathbf{1 6 5}<-\mathbf{1 9 0}$ & $\mathbf{1 9 0 < - 2 1 0}$ & $\mathbf{2 1 0 < - 2 4 0}$ & $\mathbf{2 4 0 < - 2 7 0}$ & $\mathbf{2 7 0}$ \\
\hline $\mathbf{9 5 \%}$ & 107.50 & 114.00 & 115.00 & 135.00 & 145.00 & 155.00 & 135.00 & 167.50 \\
\hline $\mathbf{9 0 \%}$ & 95.00 & 95.00 & 115.00 & 115.00 & 135.00 & 150.00 & 135.00 & 147.00 \\
\hline $\mathbf{8 0 \%}$ & 80.00 & 95.00 & 105.00 & 115.00 & 125.00 & 135.00 & 116.00 & 135.00 \\
\hline $\mathbf{7 0 \%}$ & 75.00 & 95.00 & 95.00 & 105.00 & 115.00 & 115.00 & 115.00 & 135.00 \\
\hline $\mathbf{6 0 \%}$ & 75.00 & 85.00 & 95.00 & 95.00 & 105.00 & 105.00 & 115.00 & 115.00 \\
\hline $\mathbf{5 0 \%}$ & 70.00 & 80.00 & 90.00 & 95.00 & 100.00 & 95.00 & 105.00 & 115.00 \\
\hline $\mathbf{4 0 \%}$ & 65.00 & 75.00 & 85.00 & 95.00 & 95.00 & 95.00 & 95.00 & 109.00 \\
\hline $\mathbf{3 0 \%}$ & 65.00 & 65.00 & 75.00 & 85.00 & 95.00 & 95.00 & 95.00 & 95.00 \\
\hline $\mathbf{2 0 \%}$ & 65.00 & 65.00 & 75.00 & 75.00 & 85.00 & 75.00 & 85.00 & 78.00 \\
\hline $\mathbf{1 0 \%}$ & 55.00 & 55.00 & 65.00 & 65.00 & 75.00 & 65.00 & 65.00 & 65.00 \\
\hline $\mathbf{5 \%}$ & 52.50 & 50.00 & 56.25 & 65.00 & 65.00 & 65.00 & 65.00 & 58.00 \\
\hline $\mathbf{n}$ & 49 & 99 & 184 & 294 & 171 & 144 & 63 & 45 \\
\hline Mean & 72.69 & 79.79 & 87.96 & 94.27 & 104.09 & 104.03 & 103.33 & 111.67 \\
\hline $\mathbf{S D}$ & 14.81 & 17.36 & 18.72 & 21.39 & 24.56 & 27.94 & 23.38 & 30.82 \\
\hline
\end{tabular}

Table 10. Percentile ranks and descriptive statistics for Seated Front Press Post-Training

\begin{tabular}{|c|c|c|c|c|c|c|c|c|}
\hline \multicolumn{7}{|c|}{ Body Weight Class (Ibs) } \\
\hline Percentile & $\mathbf{< 1 3 5}$ & $\mathbf{1 3 5 < - 1 5 0}$ & $\mathbf{1 5 0 < - 1 6 5}$ & $\mathbf{1 6 5 < - 1 9 0}$ & $\mathbf{1 9 0 < - 2 1 0}$ & $\mathbf{2 1 0 < - 2 4 0}$ & $\mathbf{2 4 0 < - 2 7 0}$ & $\boldsymbol{> 2 7 0}$ \\
\hline $\mathbf{9 5 \%}$ & 115.00 & 125.00 & 135.00 & 151.25 & 157.00 & 165.00 & 165.00 & 193.25 \\
\hline $\mathbf{9 0 \%}$ & 110.00 & 115.00 & 125.00 & 135.00 & 147.00 & 155.00 & 155.00 & 181.50 \\
\hline $\mathbf{8 0 \%}$ & 100.00 & 105.00 & 120.00 & 125.00 & 135.00 & 145.00 & 135.00 & 155.00 \\
\hline $\mathbf{7 0 \%}$ & 95.00 & 95.00 & 115.00 & 115.00 & 135.00 & 135.00 & 127.00 & 145.00 \\
\hline $\mathbf{6 0 \%}$ & 95.00 & 95.00 & 105.00 & 115.00 & 125.00 & 135.00 & 121.00 & 145.00 \\
\hline $\mathbf{5 0 \%}$ & 82.50 & 95.00 & 100.00 & 115.00 & 115.00 & 115.00 & 115.00 & 135.00 \\
\hline $\mathbf{4 0 \%}$ & 75.00 & 85.00 & 95.00 & 105.00 & 110.00 & 115.00 & 107.00 & 129.00 \\
\hline $\mathbf{3 0 \%}$ & 72.50 & 85.00 & 95.00 & 95.00 & 105.00 & 105.00 & 105.00 & 115.00 \\
\hline $\mathbf{2 0 \%}$ & 65.00 & 75.00 & 85.00 & 95.00 & 95.00 & 100.00 & 96.40 & 115.00 \\
\hline $\mathbf{1 0 \%}$ & 65.00 & 75.00 & 75.00 & 85.00 & 88.00 & 90.00 & 88.00 & 98.50 \\
\hline $\mathbf{5 \%}$ & 58.75 & 65.00 & 70.00 & 75.00 & 75.00 & 85.00 & 85.00 & 88.50 \\
\hline $\mathbf{n}$ & 34 & 89 & 169 & 314 & 155 & 149 & 65 & 46 \\
\hline Mean & 83.97 & 92.25 & 102.63 & 110.79 & 118.19 & 121.91 & 118.34 & 135.33 \\
\hline SD & 16.87 & 17.93 & 20.10 & 21.32 & 26.23 & 26.17 & 25.05 & 29.63 \\
\hline
\end{tabular}

\section{Index to Performance Rankings}

Excellent $=$ highest $5 \%$, Good $=$ next highest 15\%, Average $=$ middle 60\%,

Below average=next lowest $15 \%$, and Poor=lowest $5 \%$ 
Table 11. Percentile ranks and descriptive statistics for Preacher Curl Pre-Training

\begin{tabular}{|c|c|c|c|c|c|c|c|c|}
\hline \multicolumn{9}{|c|}{ Body Weight Class (Ibs) } \\
\hline Percentile & $<135$ & $135<-150$ & $150<-165$ & $165<-190$ & $190<-210$ & $210<-240$ & $240<-270$ & $>270$ \\
\hline $95 \%$ & 70.00 & 74.50 & 80.00 & 90.00 & 96.25 & 103.50 & 103.50 & 110.00 \\
\hline $90 \%$ & 70.00 & 70.00 & 75.00 & 85.00 & 90.00 & 90.00 & 95.00 & 110.00 \\
\hline $80 \%$ & 64.00 & 70.00 & 70.00 & 75.00 & 80.00 & 80.00 & 85.00 & 90.00 \\
\hline $70 \%$ & 58.50 & 60.00 & 70.00 & 70.00 & 75.00 & 75.00 & 75.00 & 79.50 \\
\hline $60 \%$ & 50.00 & 60.00 & 65.00 & 70.00 & 75.00 & 70.00 & 74.00 & 75.00 \\
\hline $50 \%$ & 50.00 & 55.00 & 60.00 & 65.00 & 70.00 & 70.00 & 70.00 & 70.00 \\
\hline $40 \%$ & 50.00 & 50.00 & 60.00 & 60.00 & 70.00 & 65.00 & 70.00 & 70.00 \\
\hline $30 \%$ & 41.50 & 50.00 & 55.00 & 60.00 & 62.50 & 60.00 & 64.50 & 70.00 \\
\hline $20 \%$ & 40.00 & 45.00 & 50.00 & 50.00 & 60.00 & 60.00 & 60.00 & 60.00 \\
\hline $10 \%$ & 40.00 & 40.00 & 40.00 & 45.00 & 50.00 & 50.00 & 50.00 & 45.00 \\
\hline $5 \%$ & 30.00 & 40.00 & 40.00 & 40.00 & 45.00 & 41.50 & 40.00 & 41.75 \\
\hline $\mathbf{n}$ & 50 & 101 & 185 & 295 & 174 & 145 & 62 & 46 \\
\hline Mean & 50.90 & 56.39 & 60.65 & 64.86 & 70.83 & 69.90 & 70.89 & 73.48 \\
\hline SD & 11.64 & 11.18 & 13.33 & 16.13 & 14.48 & 16.70 & 16.11 & 19.32 \\
\hline
\end{tabular}

Table 12. Percentile ranks and descriptive statistics for Preacher Curl Post-Training

\begin{tabular}{|c|c|c|c|c|c|c|c|c|}
\hline \multicolumn{10}{|c|}{ Body Weight Class (Ibs) } \\
\hline Percentile & $\mathbf{< 1 3 5}$ & $\mathbf{1 3 5 < - 1 5 0}$ & $\mathbf{1 5 0}<-\mathbf{1 6 5}$ & $\mathbf{1 6 5}<-\mathbf{1 9 0}$ & $\mathbf{1 9 0 < - 2 1 0}$ & $\mathbf{2 1 0 < - 2 4 0}$ & $\mathbf{2 4 0 < - 2 7 0}$ & $\mathbf{2 7 0}$ \\
\hline $\mathbf{9 5 \%}$ & 95.00 & 90.00 & 100.00 & 110.00 & 120.00 & 110.00 & 110.00 & 120.00 \\
\hline $\mathbf{9 0 \%}$ & 82.50 & 85.00 & 95.00 & 100.00 & 100.00 & 105.00 & 100.00 & 115.00 \\
\hline $\mathbf{8 0 \%}$ & 80.00 & 80.00 & 90.00 & 90.00 & 95.00 & 95.00 & 90.00 & 110.00 \\
\hline $\mathbf{7 0 \%}$ & 77.50 & 75.00 & 85.00 & 90.00 & 90.00 & 90.00 & 90.00 & 100.00 \\
\hline $\mathbf{6 0 \%}$ & 65.00 & 70.00 & 80.00 & 80.00 & 90.00 & 85.00 & 85.00 & 95.00 \\
\hline $\mathbf{5 0 \%}$ & 60.00 & 70.00 & 75.00 & 80.00 & 80.00 & 80.00 & 80.00 & 95.00 \\
\hline $\mathbf{4 0 \%}$ & 60.00 & 65.00 & 70.00 & 75.00 & 80.00 & 80.00 & 80.00 & 90.00 \\
\hline $\mathbf{3 0 \%}$ & 57.50 & 60.00 & 70.00 & 70.00 & 75.00 & 75.00 & 75.00 & 80.00 \\
\hline $\mathbf{2 0 \%}$ & 50.00 & 55.00 & 60.00 & 65.00 & 70.00 & 70.00 & 70.00 & 70.00 \\
\hline $\mathbf{1 0 \%}$ & 50.00 & 50.00 & 60.00 & 60.00 & 65.00 & 65.00 & 65.00 & 70.00 \\
\hline $\mathbf{5 \%}$ & 47.50 & 50.00 & 50.00 & 55.00 & 60.00 & 60.00 & 56.50 & 57.50 \\
\hline $\mathbf{n}$ & 34 & 90 & 176 & 324 & 158 & 149 & 65 & 49 \\
\hline Mean & 65.59 & 67.89 & 75.20 & 79.20 & 83.10 & 83.62 & 82.15 & 92.24 \\
\hline SD & 14.86 & 12.29 & 14.81 & 15.84 & 15.86 & 15.08 & 13.86 & 20.54 \\
\hline
\end{tabular}

\section{Index to Performance Rankings}

Excellent $=$ highest $5 \%$, Good $=$ next highest 15\%, Average $=$ middle 60\%,

Below average=next lowest 15\%, and Poor=lowest 5\% 
Table 13. Percentile ranks and descriptive statistics for Leg Press Pre-Training

\begin{tabular}{|c|c|c|c|c|c|c|c|c|}
\hline \multicolumn{10}{|c|}{ Body Weight Class (lbs) } \\
\hline Percentile & $\mathbf{< 1 3 5}$ & $\mathbf{1 3 5}<-\mathbf{1 5 0}$ & $\mathbf{1 5 0}<-\mathbf{1 6 5}$ & $\mathbf{1 6 5}<-\mathbf{1 9 0}$ & $\mathbf{1 9 0 < - 2 1 0}$ & $\mathbf{2 1 0 < - 2 4 0}$ & $\mathbf{2 4 0 < - 2 7 0}$ & $>\mathbf{2 7 0}$ \\
\hline $\mathbf{9 5 \%}$ & 348.50 & 405.00 & 483.00 & 495.50 & 585.00 & 585.00 & 675.00 & 755.50 \\
\hline $\mathbf{9 0 \%}$ & 295.00 & 363.50 & 419.00 & 494.20 & 542.00 & 540.00 & 643.00 & 675.00 \\
\hline $\mathbf{8 0 \%}$ & 283.00 & 315.00 & 405.00 & 405.00 & 494.80 & 495.00 & 585.00 & 585.00 \\
\hline $\mathbf{7 0 \%}$ & 228.50 & 315.00 & 350.00 & 395.00 & 425.00 & 445.00 & 495.00 & 585.00 \\
\hline $\mathbf{6 0 \%}$ & 225.00 & 295.00 & 315.00 & 335.00 & 405.00 & 405.00 & 460.00 & 521.00 \\
\hline $\mathbf{5 0 \%}$ & 225.00 & 270.00 & 315.00 & 315.00 & 385.00 & 405.00 & 405.00 & 495.00 \\
\hline $\mathbf{4 0 \%}$ & 205.00 & 230.00 & 275.00 & 315.00 & 315.00 & 360.00 & 405.00 & 407.00 \\
\hline $\mathbf{3 0 \%}$ & 181.50 & 225.00 & 245.00 & 302.00 & 315.00 & 315.00 & 373.00 & 405.00 \\
\hline $\mathbf{2 0 \%}$ & 139.00 & 225.00 & 225.00 & 270.00 & 280.00 & 315.00 & 335.00 & 328.00 \\
\hline $\mathbf{1 0 \%}$ & 135.00 & 189.50 & 223.00 & 225.00 & 225.00 & 245.00 & 315.00 & 273.00 \\
\hline $\mathbf{5 \%}$ & 130.50 & 135.00 & 181.50 & 184.50 & 225.00 & 225.00 & 225.00 & 211.00 \\
\hline $\mathbf{n}$ & 50 & 102 & 185 & 297 & 175 & 145 & 63 & 45 \\
\hline Mean & 218.00 & 269.85 & 308.79 & 335.70 & 380.54 & 393.66 & 442.22 & 480.00 \\
\hline $\mathbf{S D}$ & 65.87 & 73.84 & 89.99 & 95.88 & 113.45 & 107.96 & 129.02 & 157.71 \\
\hline
\end{tabular}

Table 14. Percentile ranks and descriptive statistics for Leg Press Post-Training

\begin{tabular}{|c|c|c|c|c|c|c|c|c|}
\hline \multicolumn{7}{|c|}{ Body Weight Class (Ibs) } \\
\hline Percentile & $<\mathbf{1 3 5}$ & $\mathbf{1 3 5 < - 1 5 0}$ & $\mathbf{1 5 0 < - 1 6 5}$ & $\mathbf{1 6 5 < - 1 9 0}$ & $\mathbf{1 9 0 < - 2 1 0}$ & $\mathbf{2 1 0 < - 2 4 0}$ & $\mathbf{2 4 0 < - 2 7 0}$ & $\boldsymbol{\mathbf { 2 7 0 }}$ \\
\hline $\mathbf{9 5 \%}$ & 433.00 & 508.50 & 585.00 & 605.00 & 675.50 & 696.00 & 695.00 & 817.75 \\
\hline $\mathbf{9 0 \%}$ & 386.00 & 455.00 & 500.00 & 545.00 & 605.50 & 635.00 & 636.50 & 769.50 \\
\hline $\mathbf{8 0 \%}$ & 359.00 & 405.00 & 468.00 & 505.00 & 585.00 & 590.00 & 598.00 & 705.00 \\
\hline $\mathbf{7 0 \%}$ & 318.00 & 405.00 & 454.50 & 495.00 & 546.50 & 585.00 & 585.00 & 675.00 \\
\hline $\mathbf{6 0 \%}$ & 315.00 & 400.00 & 420.00 & 455.00 & 517.00 & 579.00 & 560.00 & 663.00 \\
\hline $\mathbf{5 0 \%}$ & 315.00 & 365.00 & 405.00 & 425.00 & 500.00 & 520.00 & 505.00 & 590.00 \\
\hline $\mathbf{4 0 \%}$ & 300.00 & 335.00 & 405.00 & 408.00 & 488.00 & 500.00 & 495.00 & 585.00 \\
\hline $\mathbf{3 0 \%}$ & 271.00 & 315.00 & 365.00 & 405.00 & 428.50 & 467.00 & 457.00 & 571.00 \\
\hline $\mathbf{2 0 \%}$ & 225.00 & 315.00 & 315.00 & 369.00 & 405.00 & 408.00 & 407.00 & 503.00 \\
\hline $\mathbf{1 0 \%}$ & 225.00 & 271.50 & 292.00 & 315.00 & 404.00 & 379.00 & 388.50 & 405.00 \\
\hline $\mathbf{5 \%}$ & 204.00 & 228.25 & 245.00 & 315.00 & 359.75 & 315.00 & 370.25 & 366.50 \\
\hline $\mathbf{n}$ & 35 & 92 & 176 & 318 & 158 & 147 & 66 & 48 \\
\hline Mean & 304.71 & 363.97 & 405.20 & 438.86 & 502.31 & 523.64 & 519.62 & 609.79 \\
\hline SD & 69.32 & 76.05 & 95.85 & 86.37 & 98.97 & 111.68 & 102.76 & 150.88 \\
\hline
\end{tabular}

\section{Index to Performance Rankings}

Excellent $=$ highest $5 \%$, Good $=$ next highest $15 \%$, Average $=$ middle $60 \%$,

Below average=next lowest 15\%, and Poor=lowest $5 \%$ 


\section{DISCUSSION}

This study aimed to develop normative 10RM strength measures for healthy college-age males. Our data is based on a large sample of 1095 subjects, is separated into eight weight classes, and percentile ranks. The data was separated into meaningful weight categories for ease of interpretation and application. These results are important because they provide the first published 10RM normative values and performance rankings for common strength exercises for healthy college-age males.

Although other studies have generated mean or normative data for grip strength $(1,17)$, relative strength measures for bench press $(9,16)$, muscle endurance for push-ups (1), no current literature is available for 10RM normative data. Prior research has produced strength ratios based upon bodyweight and 1RM loads lifted (17) and equations to estimate RM loads $(6,10,21,22)$. However, the 10RM normative data from the present study offers a quick reference guide for practitioners. While the estimations of loads or strength ratios have their place in testing and training applications, the use of normative data comparison is an easier way to assess 10RM strength measures.

Another positive aspect of the present study is that it spans from novice to intermediate training status. The ACSM definition of a novice trainer is someone who possesses less than several years of resistance training experience (3) while NSCA defines a novice or beginner as a person who has not been trained or has just begun resistance training with less than 2 months of experience (14) or zero to six months of training (8). The normative data developed from this study is useful for both the novice, according to the ACSM definition or the beginner to intermediate level individual, according to the NSCA definitions. The performance ratings provides the lifter and coaches with feedback on strength levels, helps establish training goals, aids in modifying training programs, and provides objective data for lifter tracking of progress.

Based upon this data expected strength gains for a beginner can be estimated as they progress over a 12-week period of consistent training. The present data indicates that a typical percentage increase of strength is between 10-15\% for upper body exercises, and almost $20 \%$ for lower body exercises. While this may vary based upon physical maturity, other sport background, genetics, hormonal levels, etc., it helps with making realistic predictions of strength based upon starting levels. The ability to continue making similar percentage increases remains to be studied. While further improvements are likely it would be unwise to expect these kinds of strength gains after a lifter has reached the intermediate level of training and reaches the level of an advanced lifter.

When beginning a training program, it is common to find recommendations for starting at $8-12 \mathrm{RM}$ or 10RM loads $(3,8,14)$ but without any form of normative data a lifter is left without a point of reference for standards of performance. The tables developed from this data give the practitioner a means of comparing current 10RM loads to lifters of similar age, gender, and training status. Being the first study to establish $10 \mathrm{RM}$ normative data is useful to help determine levels of performance for the novice lifter, based upon pre-training findings (tables $5,7,9,10,12$ ) as well as after 12 weeks of structured strength training.

In addition to the application of $10 \mathrm{RM}$ normative data to training program design the data is also of value to many occupations. Training someone for a given occupation to perform job-specific tasks, in the most optimal manner and with appropriate loads, could reduce the chance of training related injuries due to overexertion. Although maximal lifts may be needed in some occupations, there is typically a physical exertion demand level that follows the U.S. Bureau of Labor Statistics that is complemented with the employer's job description. Pushing, pulling, lifting, and carrying are occupational specific tasks that are performed during work shifts requiring either maximal or repetitive displays of strength. The physical fitness assessment of tactical occupations has testing to be accepted into and graduate from training (e.g. U.S. Army basic training). Although the strength tests can vary from dynamic (e.g. pull-up), isometric (e.g. hipleg dynamometer), to isokinetic (e.g. knee extension) these all can provide an assessment that correlates to some occupational task performance $(7,11)$. The use of dynamic strength tests may have a greater application to occupational task performance thus testing a person's 10RM capabilities can be used a way to classify a person's strength level prior to their entry to training. Testing a person to meet a criterion for a job provides only an assessment of what they are physically capable of for a single attempt or time. Using the 10RM as a testing method on subsequent days provides a more robust assessment of the person's capabilities of having to repeat strength tasks that are experienced in daily work shifts.

The preparation for testing a single maximal effort is 
not necessarily practical, while 10RM are commonly used in training and would be more practical and efficient to occupational task assessment. Daily tasks are not always performed at a single maximal strength output (e.g. 100\% 1RM), but these occupational tasks are instead repeated strength actions with submaximal loads performed throughout a typical day. Testing a person's 10RM on subsequent training days provides a more robust assessment of the person's capabilities of having to repeat daily tasks. The ability to rank a person's repeated strength, based on a 10RM, can provide practitioners the ability to develop the most effective plan and programs to address physical abilities that may need improvement or others to be maintained for work, sport, or recreation.

\section{PRACTICAL APPLICATIONS}

The application of 10RM training has a long history and is a convenient, safe, and effective method for beginners undertaking a strength training program. Evaluation of strength is a common concern of lifters, athletes, coaches, and trainers. Due to the recommendations that novice lifters begin training programs with 10RM loads it is beneficial to have a reference point to a lifter's current strength levels for realistic comparisons and programming

The use of normative data for evaluation is only useful if it matches the sample of reference. The specific pre-test data provided in the present study will be useful for assessment of novice college-age males. The post-test data in the present study will be useful for application to the novice or intermediate lifter with 12 weeks of training experience. In both cases, some may choose to use the percentile rankings or performance ratings to establish a lifter profile. This kind of profiling can be useful for lifter grading, ranking, and programming.

\section{REFERENCES}

1. Acevedo EO, Starks MA. Exercise testing and prescription lab manual. Champaign IL: Human Kinetics, 2011.

2. American College of Sports Medicine. ACSM's guidelines for exercise testing and prescription. Lippincott Williams \& Wilkins, 2018.

3. American College of Sports Medicine. American College of Sports Medicine position stand. Progression models in resistance training for healthy adults. Med Sci Sports Exerc, 41, 687-708, 2009.

4. Baechle TR, Earle RW. Resistance Training and Spotting Techniques. In: Essentials of Strength Training and Conditioning 3rd ed, TR Baechle and RW Earle, eds
Champaign, IL: Human Kinetics, 2008

5. Brill PA, Macera CA, Davis DR, Blair SN, Gordon N. Muscular strength and physical function. Med Sci Sports Exerc, 32: 412, 2000.

6. Brzycki M. Strength testing: Predicting a one-rep max from reps-to-failure. J Phys Educ Recreat Dance 64: 88-90, 1993.

7. Canetti EFD, Dawes JJ, Drysdale PH, et al. Relationship between metabolic fitness and performance in police occupational tasks. J Sci Sport Exer https://doi. org/10.1007/s42978-020-00066-12020.

8. Coburn JW, Malek MH. NSCA's Essentials of Personal Training 2nd ed. Champaign, IL: Human Kinetics, 2012.

9. The Cooper Institute. The Physical Fitness Specialist Manual. The Cooper Institute for Aerobics Research, Dallas, 1997.

10. Epley B. Boyd Epley Workout. Lincoln: University of Nebraska, 1985.

11. Marins EF, Cabistany L, Bartel C, Dawes JJ, Del Vecchio FB. Effects of personal protective equipment on metabolism and performance during an occupational physical ability test for federal highway police officers. The Journal of Strength \& Conditioning Research, 34(4), pp. 1093-1102. 2020.

12. Gibson AL, Wagner D, Heyward V. Advanced Fitness Assessment and Exercise Prescription, 8E. Human kinetics, 2018.

13. Gotshalk LA, Loebel CC, Nind BC, et al. Hormonal responses of multiset versus single-set heavy-resistance exercise protocols. Can J Appl Physiol, 22, 244-255, 1997.

14. Caulfield S, Berninger, D. Exercise Technique for Free Weight and Machine Training. In: Essentials of Strength Training and Conditioning. 4th ed. GG Haff and NT Triplett, eds. Champaign, IL: Human Kinetics, 2016.

15. Herman-Montemayor JR, Hikida RS, Staron RS. Earlyphase satellite cell and myonuclear domain adaptations to slow-speed vs traditional resistance training programs. J Strength Cond R, 29, 3105-3114, 2015.

16. Heyward $\mathrm{VH}$. Advanced Fitness Assessment and Exercise Prescription. Champaign, IL: Human Kinetics, 2002.

17. Hoffman J. Norms for fitness, performance, and health. Champaign, IL: Human Kinetics, 2006

18. Izquierdo M, Ibanez J, González-Badillo JJ, et al. Differential effects of strength training leading to failure versus not to failure on hormonal responses, strength, and muscle power gains. J Appl Physiol, 100, 1647-1656, 2006.

19. Jurca R, Lamonte MJ, Barlow CE, et al. Association of muscular strength with incidence of metabolic syndrome in men. Med Sci Sports Exerc, 37: 1849, 2005.

20. Jurca R, Lamonte MJ, Church TS, et al. Associations of muscle strength and fitness with metabolic syndrome in men. Med Sci Sports Exerc, 36: 1301-1307, 2004.

21. Kuramoto AK, Payne VG. Predicting muscular strength in women: A preliminary study. Res Q Exerc Sport, 66: 168172, 1995

22. Lander J. Maximum based on reps. NSCA Journal, 6: 6061, 1984

23. Morton RW, Oikawa SY, Wavell CG, et al. Neither load nor systemic hormones determine resistance trainingmediated hypertrophy or strength gains in resistancetrained young men. J Appl Physiol, 121: 129-138, 2016.

24. Newman AB, Kupelian V, Visser M, et al. Strength, but not muscle mass, is associated with mortality in the health, aging and body composition study cohort. J Gerentol A Biol Sci Med Sci, 61: 72-77, 2006.

25. Ratamess NA, Alvar BA, Evetoch TK, et al. Progression 
Models in Resistance Training for Healthy Adults. Med Sci Sports Exerc, 41: 687-708, 2009.

26. Reiman MP, Manske RC. Functional testing in human performance. Champaign, IL: Human kinetics, 2009.

27. Saint-Maurice PF, Laurson KR, Kaj M, Csányi T.

Establishing normative reference values for standing broad jump among Hungarian youth. Res Q Exerc Sport, 86: S37-S44, 2015.

28. Saint Romain B, Mahar MT. Norm-referenced and criterion-referenced reliability of the push-up and modified pull-up. Meas Phys Educ Exerc Sci, 5: 67-80, 2001.

29. SPSS (2018). How does SPSS calculate percentiles in FREQUENCIES or CTABLES? https://www.ibm.com/ support/pages/how-does-spss-statistics-calculatepercentiles-frequencies-or-ctables

30. Sterkowicz-Przybycien, K. L., \& Fukuda, D. H. (2014). Establishing normative data for the special judo fitness test in female athletes using systematic review and metaanalysis. The Journal of Strength \& Conditioning Research, 28(12), 3585-3593.

31. Stone MH. Position statement: explosive exercises and training. Natl Strength Cond Assoc J, 15:7-15, 1993. 